\title{
Can Additional Information really have Negative Welfare Effects? - A Note on ARROW's Risk Allocation and Information
}

\author{
by Friedrich Breyer*
}

In a recent lecture on "Risk Allocation and Information", ARROW [1978, p. 9] maintains that "an increase in information may lower the efficiency of the market" in that it "effectively prevents the sharing of risk-bearing and destroys the corresponding utility gain" (ibid.). This result is indeed surprising, but it depends, as we shall argue below, on a questionable usage of the concepts of "ex ante" probability and expected utility. For a proof of his assertion cited above, ARROW considers the following example: There are two individuals, each endowed with a stock of one unit of a particular commodity : one (Walras) has a unit of wheat, the other (Böhm-Bawerk) a unit of barley. There are two weather states with probability $\frac{1}{2}$ each : in state $W$, all the barley is destroyed and none of the wheat, in $B$ all the wheat is destroyed and none of the barley. Each of the individuals is assumed to have a von Neumann utility function $u=\log y$, where $y$ is the total amount of wheat and barley consumed by him. ARROW shows that with perfect contingent markets and no further information the equilibrium consists of the following contract: Walras sells to Böhm-Bawerk $\frac{1}{2}$ unit of wheat-if- $W$ and buys from him $\frac{1}{2}$ unit of barley-if- $B$. The expected utilities of each of them are, therefore

$$
E\left(u_{\mathrm{W}}\right)=E\left(u_{B}\right)=\frac{1}{2} \log \frac{1}{2}+\frac{1}{2} \log \frac{1}{2}=\log \frac{1}{2},
$$

the certainty-equivalent income are $y_{\mathrm{W}} *=y_{B}{ }^{*}=\frac{1}{2} \cdot 1$

Now ARROW introduces into his model the occurence of additional public information : Both individuals are informed which weather state will prevail. If it is state $W$, Böhm-Bawerk loses his purchasing power, and no transaction takes place. Walras' consumption is $y_{\mathrm{W}}=1$, his utility $u_{\mathrm{W}}=0$, Böhm-Bawerk's consumption $y_{B}=0$, his utility $u_{B}=-\infty$. If $B$ is known to prevail, the allocation is reversed.

* University of Heidelberg.

$1 y_{i}^{*}$ is computed by setting $u\left(y_{i}^{*}\right)=E\left(u_{i}\right), \quad i=W, B$. 
This exactly is the point of departure for ARROW's conclusion cited above, for he argues: " $E x$ ante, states $W$ and $B$ occur with probability $\frac{1}{2}$ each; hence, for each individual, the expected utility is $-\infty$, and therefore [his certainty-equivalent consumption, F.B.] $y_{\mathrm{W}}=y_{B}=0 .{ }^{2}$ In our view, this argument is based on a fallacy : Admittedly, once the additional information is dispersed, the potential gains from trade are destroyed, but if ARROW mentions ex ante probabilities there must be a time period prior to the dissemination of the knowledge which weather state will prevail. Let $t_{0}$ be the starting point of the analysis: In $t_{0}$, both individuals hold the ex-ante (subjective) probabilities $p_{\mathrm{W}}=p_{B}=\frac{1}{2}$ for the states $W$ and $B$. Let further $t_{1}$ be the moment of the arrival of the new information, and $t_{2}$ the time of the occurrence of the weather itself. Clearly, $t_{0} \leqslant t_{1}<t_{2}$ must hold to make the information relevant. Let us first assume $t_{0}<t_{1}<t_{2}$ (see Figure 1).

Figure 1

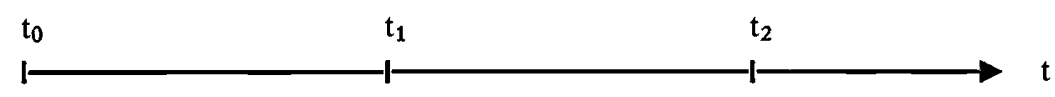

In this case, both individuals should consider the arrival of (possibly bad) news rather than the weather state itself as the risk against which one should insure oneself. For, as long as there are no production possibilities, there is no logical distinction between a certain knowledge that a particular event will occur and the event itself.

In the initial state between $t_{0}$ and $t_{1}$, the expected utilities given by ARROW, viz. $E(u \mathrm{w})=E\left(u_{B}\right)=-\infty$, apply only if Walras and Böhm-Bawerk fail to sign a contract on contingent commodities within this time period. Otherwise, they are $E\left(u_{\mathrm{W}}\right)=E\left(u_{B}\right)=\log \frac{1}{2}$ (just like in the case of no information). If rational behavior is assumed, they will clearly strike their bargain as soon as possible after $t_{0}$, at least if the time of the risky event "additional information", $t_{1}$, is unknown to them. The opportunity to shift risks by trading contingent commodities vanishes only in the extreme case that the additional information is known from the beginning on, that is if $t_{1}$ equals $t_{0}$. But in this case it is misleading to say, as ARROW does, that "ex ante, states $W$ and $B$ occur with probability $\frac{1}{2}$ each" (p. 9). At least under the subjective probability concept, which is explicitly employed by ARROW (cf. p. 6), probabilities and expected utilities have to refer to a particular point or period of time in which somebody holds these expectations. With $t_{0}=t_{1}$ such a time span does not exist. If there is a-priori knowledge that state $W$ occurs, the expected utilities are rather

$$
2 E(u)=\frac{1}{2} u(1)+\frac{1}{2} u(O)=\frac{1}{2} \cdot O+\frac{1}{2} \cdot(-\infty)=-\infty
$$


$E\left(u_{\mathrm{W}}\right)=0, E\left(u_{B}\right)=-\infty$, otherwise $E\left(u_{\mathrm{W}}\right)=-\infty, E\left(u_{B}\right)=0$. Neither of these outcomes can be compared with the no-information outcome (1) by using the Pareto criterion. Consequently the availability of a-priori information may be said to have distributional rather than welfare effects.

Another remark seems in order : According to ARROW (p. 9), the harmful effect of information on the efficiency of the market was first noted by HIRSHLEIFER (1971). This seems to be a misinterpretation, since HIRSHLEIFER did not maintain that sure information has a negative social value, but only that its social value in a pure exchange-no production case is zero (p. 564 f.). ${ }^{3}$

\section{REFERENCES}

ARROW, K. J. [1978] : "Risk allocation and information : Some recent theoretical developments", The Geneva Papers on Risk and Insurance, 8, 5-19.

HIRSHLEIFER, J. [1971]: "The private and social value of information and the reward to inventive activity", American Economic Review, 61, 561-574.

3 In another respect, however, HIRSHLEIFER's analysis, too, seems questionable : In several cases (pp. 564, 565, 566) he computes, similar to ARROW, an "expected utility given perfect information, using prior [probability] weights $\pi_{a}=\bullet 6, \pi_{b}=\bullet 4$." Probability weights and perfect information clearly contradict each other, since probabilities imply imperfect information. Any conclusions drawn from the simultaneous usage of both concepts must, therefore, be open to doubt. 